\title{
Experimental and modelling aspects of the reactive extrusion process
}

\author{
Philippe Cassagnau ${ }^{1, *}$, Véronique Bounor-Legaré ${ }^{1}$, and Bruno Vergnes ${ }^{2}$ \\ ${ }^{1}$ Université de Lyon, Ingénierie des Matériaux Polymères, UMR CNRS 5223, 69622 Villeurbanne, France \\ 2 MINES ParisTech, PSL Research University, CEMEF, UMR CNRS 7635, CS 10207, 06904 Sophia Antipolis, France
}

Received: 3 May 2019 / Accepted: 19 July 2019

\begin{abstract}
Reactive extrusion consists in using an extruder as a continuous chemical reactor. It is not a recent process, but it has been rapidly developed during the last thirty years and is more and more used today for the chemical modification of existing polymers. Among the various extrusion systems (single screw extruders, counter- and corotating twin-screw extruders, co-kneaders), the corotating twin-screw extruders are today the most widely used in reactive extrusion. After a presentation of the main advantages and drawbacks of the reactive extrusion, we will describe the way to control the process through on-line and in-line monitoring. Then, a modelling approach based on continuum mechanics will be presented, followed by an example of industrial applications of this particular process.
\end{abstract}

Keywords: Reactive processing / twin-screw extrusion / monitoring / modelling

\section{Introduction}

Much research has been devoted to the reactive processing of polymers and more particularly to the use of a twinscrew extruder (reactive extrusion process) for the industrial development of new thermoplastic materials. Technical analyzes of the reactive extrusion processes have been carried out continuously since the twin-screw extruders have received considerable attention as reactors.

In fact, one of the important advantages of the extruder over batch reactors is to facilitate the bulk reactive process in a continuous way, with high viscosity, solvent-free reactive systems. However, reactive processing combines the usual difficulties of polymer processing and the problems of controlling a chemical reaction under very specific conditions: very viscous medium $\left(\eta \approx 10^{3} \mathrm{Pas}\right)$, high temperature $\left(T \approx 250^{\circ} \mathrm{C}\right)$ and short residence time $(t \approx 1 \mathrm{~min})$. As a result, reactive processing developments require fundamental research in the fields of chemistry, rheology, flow and mixing modeling, in-line instrumentation, and process control. A number of reactive systems are today involved in reactive processing, such as, for example, chemical modification of molten polymers, bulk polymerization, reactive compatibilization of immiscible polymer blends by reaction at the interface, and in situ polymerization and/or crosslinking of one phase in a polymer blend.

\footnotetext{
* e-mail: philippe.cassagnau@univ-lyon1.fr
}

The role of diffusion and mixing is exacerbated in the case of reactive extrusion by the high viscosity of molten polymers, the eventual change in viscosity with the extent of the reaction and the short residence times. In addition, reactive processes with polymers are very complicated to design and control because several highly nonlinear and coupled phenomena are involved. For example, the flow generated by the rotation of the screws is fundamentally laminar but is difficult to simulate because of its threedimensional and non-steady state nature. Furthermore, the molten polymers are generally non-Newtonian and their properties may change along the extruder (spatial and temporal evolution) due to the mixing process and/or the evolution of the chemical reaction. In addition, the diffusion process must also be taken into account as it can become an important limiting step at the molecular scale of mixing, and as long as chemical reactions take place. Finally, heat transfer must be considered because of viscous dissipation and chemical reactions. All these transport and chemical phenomena are coupled, at least with the dependence of the transport properties (viscosities, diffusion coefficients, etc.) as a function of temperature and composition (Fig. 1).

This introductory part emphasizes the complexity of the reactive processing aspects, described for example by Beyer and Hopmann [2]. The present paper first introduces the advantages and disadvantages of the reactive extrusion process. Then, some methods for in-line and on-line monitoring of relevant data (pressure, chemical conversion, 


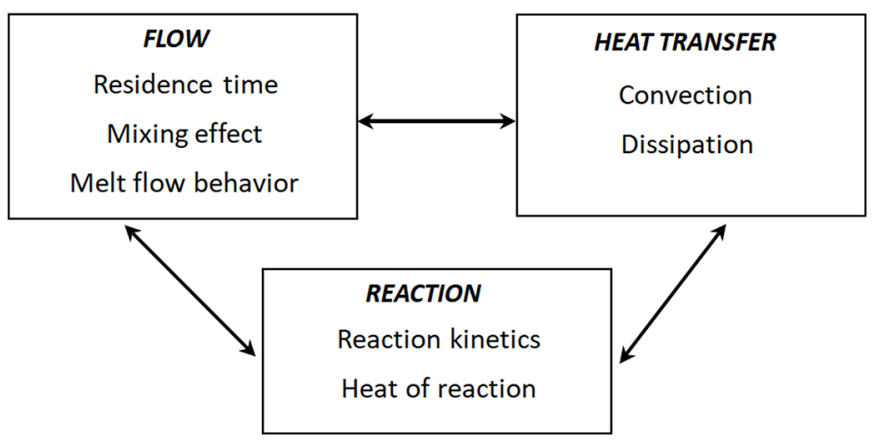

Fig. 1. Example of coupling in reactive processing (adapted from Michaeli et al. [1]).

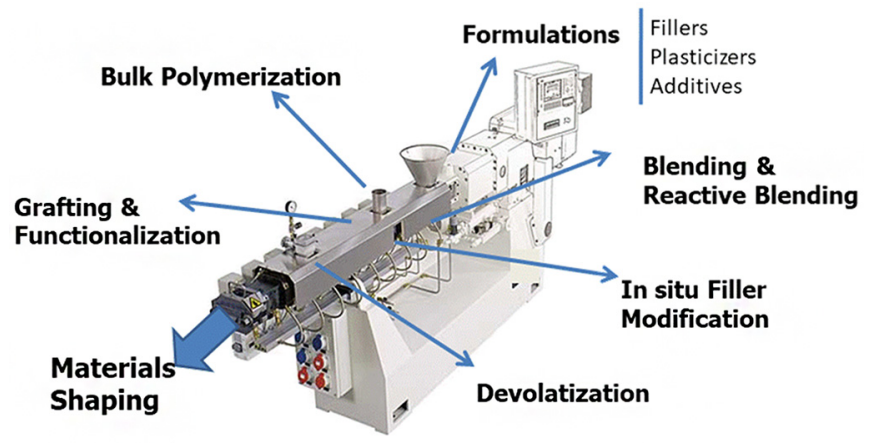

Fig. 2. Scheme of the reactive extrusion process.

residence time distribution, etc.) at the die exit are presented. Finally, this article focuses on modeling aspects and some industrial developments.

\section{Advantages and disadvantages of reactive extrusion}

As stated in the introduction, reactive extrusion is now considered to be an effective means of continuously polymerizing monomers and/or modifying polymers (Fig. 2). In particular, intermeshing co- and counterrotating twin-screw extruders have proved to be a good technical and economical solution for the reactive processing of thermoplastic polymers. The main advantages of the reactive extrusion process can be described as follows:

- polymerization and/or chemical modification conducted in the bulk, in the absence of solvents;

- efficient devolatilization, leading to effective removal of residual monomers or reaction sub-products;

- fast and continuous process;

- modular design allowing sequencing steps and the process of complex formulations (filler, plasticizer, etc.).

However, reactive extrusion also has certain disadvantages, which are in fact the counterparts of the

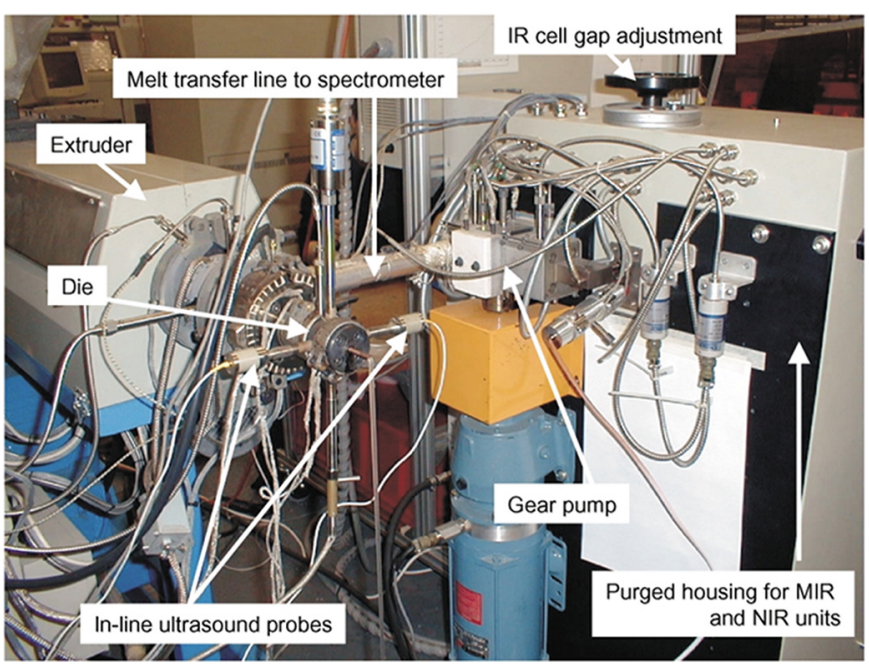

Fig. 3. In-line and on-line measurements at the exit of the extruder. Reprinted from Coates et al. [3].

main advantages:

- the high viscosity of molten polymers leads to possible strong viscous dissipation, and consequently to the developments of side reactions (thermal degradation, for example);

- the short residence time limits the reactive extrusion to fast reactions;

- complex geometry and coupled phenomena (mass and energy transfers, viscous dissipation, extent of reaction, etc.) lead to difficulties in process control;

- the scale-up of complex formulations from lab-scale to industrial extruders is still an important challenge.

\section{On-line and in-line monitoring}

In terms of industrial development, it is usually necessary to measure some relevant parameters such as residence time distribution (RTD), melt temperature, die pressure and chemical conversion. On-line and especially in-line monitoring is ideal for reactive extrusion as it eliminates the delay time associated with conventional off-line reaction monitoring techniques. In-line measurements are made using probes located in the process line, i.e. in the main flow stream.

Numerous measurement methods can be applied to polymer processing, such as spectroscopy (infrared (IR), ultraviolet (UV) and UV/fluorescence, Raman, dielectric), rheometry, optical and ultrasonic techniques. For example, Coates et al. [3] reported an exploration of the application of in-process spectroscopy (on-line mean infrared (MIR), on-line and in-line near-infrared (NIR), and in-line Raman) for process monitoring of polyethylene and polypropylene blends in a single screw extruder (Fig. 3).

From an experimental point of view, the application of such techniques to polymer melts is difficult and limited due to the high pressure and temperature (typically, $10 \mathrm{MPa}$ and $200^{\circ} \mathrm{C}$ ) encountered in polymer extrusion. However, with the improvement of the probes, notably thanks to the use of sapphire windows, and the development of optic-fiber 
spectrometers, the use of these techniques, and more specifically NIR, are becoming easier. It can also be noted that the use of optic-fiber probes for remote data collection far from the processing line (up to a few hundred meters) has often contributed to the development of robust, process-oriented NIR spectrometers. Nevertheless, the quantitative analysis of a reactive polymer medium by NIR spectroscopy is not always simple. In general, it is necessary to use chemometric methods to extract qualitative and quantitative information from NIR spectra [4].

\section{Modelling}

Polymer reactive processes are very complicated to design and control because of the need to deal with a large number of highly non-linear and coupled phenomena. In the modelling of the reactive extrusion, various strategies can be considered: the use of chemical engineering models, based on associations of ideal reactors, or models based on continuum mechanics, based on different levels of simplifications (1D, 2D and fully 3D). A simple 1D simulation approach can provide a global description of the process, from the hopper to the die exit, whereas 3D models allow an accurate local description of the flow field. However, most simulations of reactive extrusion are based on simplified steady-state 1D models, in which the residence time, temperature, and extent of the reaction are assumed to have a uniform distribution in any axial cross section of the extruder. The main advantage of 1D models is that the simulations are simple, easy to perform, and do not take time. The examples presented below were obtained using the Ludovic ${ }^{\odot}$ software, initially designed to calculate the flow conditions along a twin-screw extruder [5]. To be applied to reactive extrusion, the flow model has to be coupled with reaction kinetics and possibly a rheokinetic module, describing the change in viscosity with the extent of the reaction [6]. This simulation was successfully applied to various reactive systems: reactive blending of polymers [7], extension of polyamide 12 chains by a coupling agent [8], controlled degradation of polypropylene [9], cationization of starch [10], synthesis of nanoparticles by sol-gel method [11], transesterification reaction of EVA copolymer [12]. For example, Figure 4 shows the change in conversion rate during the polymerization of $\varepsilon$-caprolactone in a twin-screw extruder, for the same screw speed and different feed rates [13]. It appears that the conversion rate develops mainly in the blocks of kneading discs. It is lower at higher feed rate, because of the corresponding reduction in residence time. A good correlation is observed between simulated and experimental data.

Previous models only predict the steady-state behavior of the process. With regard to the goal of automatic control of reactive extrusion, a dynamic 1D model was developed by Choulak et al. [14]. This model predicts the extruder's transient (and of course, steady-state) behavior, i.e. pressure, filling ratio, monomer conversion, temperature, and residence time distribution (RTD) in various processing conditions. The model combines a global flow description with a local chemical engineering approach.

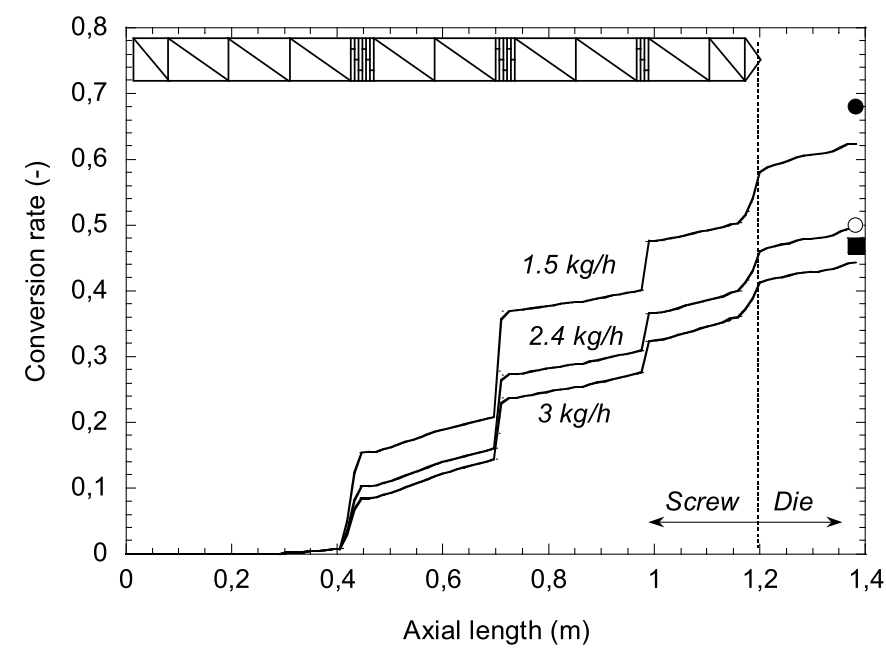

Fig. 4. Polymerization of $\varepsilon$-caprolactone; influence of feed rate on conversion rate at constant screw speed. Symbols $(\mathbf{0}: 1.5 \mathrm{~kg} / \mathrm{h}$; $\mathrm{O}: 2.4 \mathrm{~kg} / \mathrm{h}$; $: 3 \mathrm{~kg} / \mathrm{h}$ ) represent experimental measurements by 1H NMR (adapted from [13]).

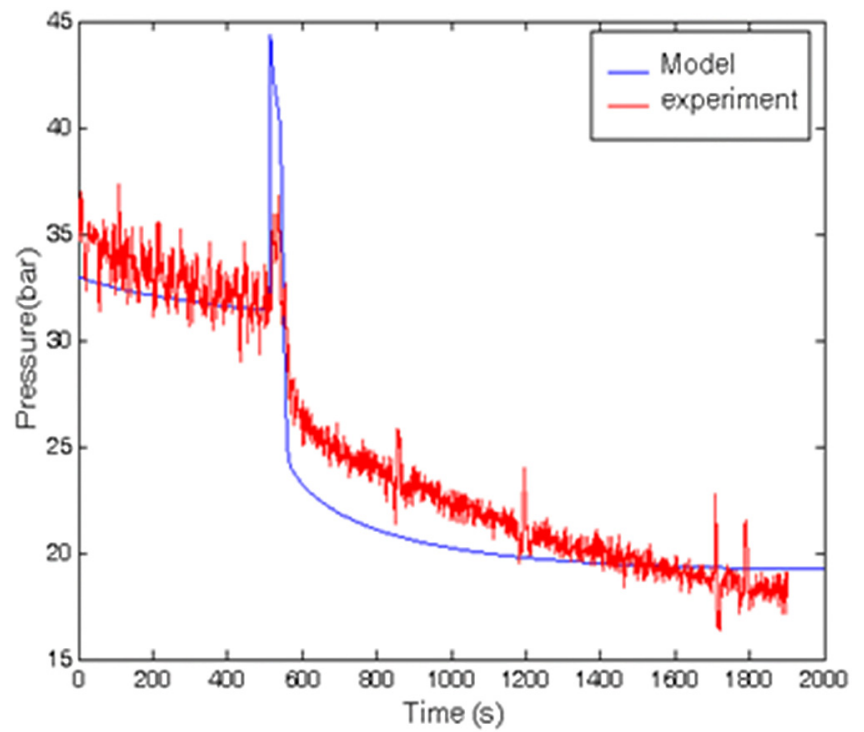

Fig. 5. Transient behavior of the pressure at the die during a step change in screw speed (from 200 to $300 \mathrm{rpm}$ ). Reprinted from Choulak et al. [14].

It consists of a cascade of perfectly stirred tank reactors that can be either fully filled with backflow or partially filled, depending on the processing conditions. A piece of barrel and screw is associated with each reactor. The material and energy balance equations are written in a differential manner to simulate the transient behavior. This model is able to reproduce the main transient and steady-state effects occurring during reactive (or nonreactive) extrusion. The experimental validation was carried out on the simulation of the pressure at the die during the polymerization of $\varepsilon$-caprolactone. As an example of result, the transient response of the pressure to a step change in the screw speed is shown in Figure 5. 


\section{Industrial applications}

In the industrial developments of many polymer formulations for new materials, reactive extrusion is generally the most viable technological and economic solution because it allows several formulation steps to be combined in terms of compatibilisation, viscosity control, purification (devolatilization of volatile organic compounds), etc. The compatibilisation of immiscible polymer blends by in situ synthesis of a copolymer at the interface during reactive extrusion is nowadays very common in the industrial world. Numerous examples can be cited, the best known of which is the PE/PA6 mixture compatibilized by the addition of $\mathrm{PE}$ grafted with maleic anhydride (PE-g-MA), leading to the formation of a copolymer at the $\mathrm{PE} / \mathrm{PA}$ interface.

\subsection{Thermoplastic Vulcanizates (TPV)}

The development of blends in which one of the two phases is generated in situ is less common, although it offers the possibility of developing new materials from a thermosetting or crosslinked phase. In this strategy, we can consider the development of Thermoplastic Vulcanizate (TPV) whose synthesis principle is the in situ crosslinking of an elastomer phase during mixing in the molten state. The best known products (Exxon Santoprene ${ }^{\Theta}$ and Hutchinson Vegaprene ${ }^{\odot}$ ) are based on the crosslinking of an EPDM phase (crosslinking with phenolic resins or radical chemistry) in a polypropylene matrix. The mechanical properties of TPV are close to those of a crosslinked elastomer, while maintaining a processing character specific to thermoplastics. In fact, reactive extrusion is the only technology that controls the dispersion of a crosslinked elastomeric phase in a thermoplastic matrix, the dispersion of previously crosslinked rubber particles leading to much less efficient properties. This concept seems relatively simple but industrial developments have often been difficult because of the complex influence of the processing conditions on the final properties. Scale-up from laboratory to industrial machines therefore requires extensive testing. To illustrate these points, the formulations of these TPV are known, but the preparation and/or shaping processes are the subject of the best kept trade secrets.

\subsection{Polyamides synthesis}

Given the short residence times in an extruder, a chemistry well suited to reactive extrusion is the cycle opening, which allows polyamides to be synthesized without creating by-products. However, it is limited to PA6, PA12 and copolymers of PA6-12, ع-caprolactam and lauryl-lactam being the two main cyclic monomers available [15].

In the case of polycondensates, reactive extrusion is used as a variant of usual synthesis processes, in particular to reduce reaction times. For example, reactive extrusion is used to obtain polyamides of high molar masses from prepolymers or oligomers, synthesized by one or more conventional reactor processes. The extrusion step, carried out under reduced pressure, allows the post-condensation of the pre-polymers introduced into the extruder. The materials synthesized in this case are generally semiaromatic or aromatic polyamides, whose preparation is less easy than that of aliphatic polyamides, in particular because of their thermal characteristics. The extruders used to carry out this molten post-condensation step of PA oligomers or pre-polymers generally have a length to diameter ratio $(L / D)$ of 20 to 30 , with a single vacuum extraction point.

Recently, Lagneaux et al. $[16,17]$ carried out in a single extrusion step the polymerization of polyamides of high or even very high molar mass, starting from the monomers, introduced separately into the extruder, without any preliminary reaction or formation of salt. The extruder used is a corotating twin-screw extruder longer than conventional ones dedicated to compounding or reactive extrusion processes. Its minimum length is more than $80 \mathrm{D}$ and can reach $110 D$. Despite this length, the reaction time corresponding to the residence time in the extruder remains very short, in the order of $3-6 \mathrm{~min}$.

\subsection{Polymer recycling}

The industrial sorting of post-consumer waste often does not provide good levels of purity for the batches to be regenerated in new materials. In particular, high density polyethylene (HDPE) and PP have similar densities and are found together at the end of the flotation sorting stages. Rather than adding an additional sorting step, for example with spectroscopic technologies, advantage can be taken of the presence of PE, which shows good impact properties, but is not miscible with $\mathrm{PP}$ and is much more viscous. To enhance these impact properties in $\mathrm{PP} / \mathrm{PE}$ mixtures, it will be essential to have good $\mathrm{PP} / \mathrm{PE}$ interfaces, which requires compatibility between the PP and PE phases, the latter being highly viscous, due to its degradation.

The relatively high viscosities of the waste materials must also be adjusted to meet current trends in Melt Flow Index (MFI) for injection molding. Thus, the molar masses of PP and therefore its viscosity can be controlled by radical degradation (chain scission reactions), for example by adding peroxides during extrusion. However, lower molar masses will necessarily lead to deterioration in impact resistance (toughness). From a formulation point of view, the addition of an elastomer phase is often the solution to improve this impact resistance.

Finally, the constraints, both in terms of hygiene and safety as well as the surface appearance of the parts, involve purifying the newly formulated materials by eliminating small hydrophilic molecules (oils, oligomers, etc.). A "water washing" during extrusion implementation will provide a relevant answer to these questions. All these aspects have been solved by two subsequent reactive extrusion steps $[18,19]$ as shown by the scheme in Figure 6 . The final product is a bumper made at the industrial scale from $\mathrm{PP}$ and PE wastes. 


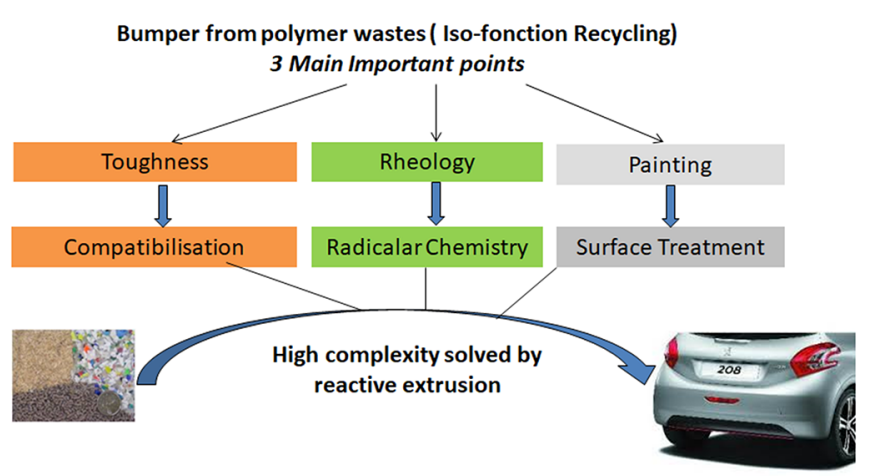

Fig. 6. Principle of the recycling of $\mathrm{PP} / \mathrm{PE}$ waste for the processing of automotive bumpers by reactive extrusion (compatibilisation, viscosity control and purification).

\section{Conclusion}

In this paper, the advantages and disadvantages of the reactive extrusion process have been introduced. Some methods for in-line and on-line monitoring of relevant data like pressure, chemical conversion, residence time have been presented. Finally, modeling aspects, both for the understanding and the optimization of the process and also for its control have been evoked. Industrial developments have been illustrated by results on the elaboration of Thermoplastic Vulcanizates, synthesis of polyamides, and polymer recycling for producing automotive bumpers.

\section{References}

[1] W. Michaeli, A. Grefenstein, U. Berghaus, Twin-screw extruders for reactive extrusion, Polym. Eng. Sci. 35, 14851504 (1995)

[2] G. Beyer, C. Hopmann, Reactive Extrusion, John Wiley, New York, 2017

[3] P.D. Coates, S.E. Barnes, M.G. Sibley, E.C. Brown, H.G.M. Edwards, I.J. Scowen, In-process vibrational spectroscopy and ultrasound measurements in polymer melt extrusion, Polymer 44, 5937-5949 (2003)

[4] D. Fischer, J. Mueller, S. Kummer, B. Kretzschmar, Real time monitoring of morphologic and mechanical properties of polymer nanocomposites during extrusion by near infrared and ultrasonic spectroscopy, Macromol. Symp. 305, 10-17 (2011)

[5] B. Vergnes, G. Della Valle, L. Delamare, A global computer software for polymer flows in corotating twin screw extruders, Polym. Eng. Sci. 38, 1781-1792 (1998)
[6] B. Vergnes, F. Berzin, Modelling of reactive systems in twin screw extrusion: challenges and applications, Comp. Rendus Chim. 9, 1409-1418 (2006)

[7] A. De Loor, P. Cassagnau, A. Michel, L. Delamare, B. Vergnes, Reactive blending in a twin-screw extruder: experimental and theoretical approaches, Int. Polym. Process. 11, 139-146 (1996)

[8] Y. Chalamet, M. Taha, F. Berzin, B. Vergnes, Carboxyl terminated polyamide 12 chain extension by reactive extrusion using a dioxazoline coupling agent. Part II: effects of extrusion conditions, Polym. Eng. Sci. 42, 2317-2327 (2002)

[9] F. Berzin, B. Vergnes, P. Dufossé, L. Delamare, Modelling of peroxide initiated controlled degradation of polypropylene in a twin screw extruder, Polym. Eng. Sci. 40, 344-356 (2000)

[10] F. Berzin, A. Tara, L. Tighzert, B. Vergnes, Computation of starch cationization performances by twin screw extrusion, Polym. Eng. Sci. 47, 112-119 (2007)

[11] W. Bahloul, O. Oddes, V. Bounor-Legaré, F. Mélis, P. Cassagnau, B. Vergnes, Reactive extrusion processing of polypropylene $/ \mathrm{TiO}_{2}$ nanocomposites by in-situ synthesis of the nanofillers, AIChE J. 57, 2174-2184 (2011)

[12] F. Berzin, B. Vergnes, Transesterification of ethylene acetate copolymer in a twin screw extruder, Int. Polym. Process. 13, 13-22 (1998)

[13] A. Poulesquen, B. Vergnes, P. Cassagnau, J. Gimenez, A. Michel, Polymerization of e-caprolactone in a twin screw extruder: experimental study and modeling, Int. Polym. Process. 16, 31 (2001)

[14] S. Choulak, F. Couenne, Y. Le Gorrec, C. Jallut, P. Cassagnau, A. Michel, Generic dynamic model for simulation and control of reactive extrusion, Ind. Eng. Chem. Res. 43, 7373-7382 (2004)

[15] S.K. Ha, J.L. White, Continuous polymerization and copolymerization of lauryl lactam in a modular corotating twin screw extruder, Int. Polym. Process. 13, 136-141 (1998)

[16] D. Lagneaux, J. Gimenez, A.C. Brosse, L. Goujard, H. Sautel, Procédé de préparation de polyamide par extrusion réactive et extrudeuse adaptée pour la mise en œuvre d'un tel procédé, French Patent 2993 887, 2016

[17] D. Lagneaux, J. Gimenez, A.C. Brosse, L. Goujard, H. Sautel, Method for preparing a polyamide by reactive extrusion, and extruder adapted for the implementation of such a method, US Patent 9,453,107, 2016

[18] F. Viot, J. Guillet, P. Cassagnau, V. Massardier-Nageotte, R. Sonnier, Method for treating a material derived from recovery and grinding, Patents FR 2008005816720081201 , WO2009FR52341 20091130, 2010

[19] F. Viot, P. Cassagnau P, F. Mélis, V. Massardier-Nageotte, R. Sonnier, Procédé de granulation de polymères et produit issu de ce procédé, Patents FR1260275A, US14/438, 137, 2012

Cite this article as: P. Cassagnau, V. Bounor-Legaré, B. Vergnes, Experimental and modelling aspects of the reactive extrusion process, Mechanics \& Industry 20, 803 (2019) 